\title{
Performance Study of MiMO Systems in a Clustered Multipath Channel
}

\author{
Guangze Zhao, Sergey Loyka \\ School of Information Technology and Engineering (SITE) \\ University of Ottawa, 161 Louis Pasteur, Ottawa, Ontario, K1N 6N5, Canada \\ Email: gzhao@site.uottawa.ca, sloyka@site.uottawa.ca
}

\begin{abstract}
Fading correlation is one of the fundamental limits on the performance of multi-antenna systems. Accurate modeling of the angular power distribution (pdf) is essential for accurate prediction of correlation. Previous studies were limited to the case of single-cluster distribution or uniform pdf. Measurements indicate that multipath often arrives in several angular clusters and with non-uniform angular cluster pdf. We begin with the popular Salz-Winters model and extend it to the case of multi-cluster channel and to the case of non-uniform cluster pdf. Generic model as well as various approximations, which result in compact closed-from expressions, are studied in detailed. It is shown that the correlation in two clusters channel has an oscillatory behavior as antenna spacing increases regardless of multipath $\mathrm{AOA}$ distributions; the envelope of correlation is determined by a single cluster angular spread while the oscillations within the envelope are determined by the inter-cluster angular spread. There exist two type of correlation zeros, which can be beneficially used in system design. The cluster angular pdf has negligible impact on the MIMO capacity and, hence, simpler uniform model can be used without degradation in accuracy.
\end{abstract}

Index Terms-Wireless propagation channel, fading correlation, MIMO capacity

\section{INTRODUCTION}

Understanding of spatial fading correlation is crucial for accurate prediction of multi-antenna (MIMO) system performance. It is well know that the fading correlation in spatially separated antennas depends on the angular distribution of the incoming multipath [1]. While some special cases of the angular pdf have been investigated [17], no detailed understanding of the problem has been achieved so far. Most studies were limited to the case of single cluster with uniform [1-3] or non-uniform pdf [4-7]. Only preliminary studies of multi-cluster channels have been reported [3-5]. It is important to understand the correlation behavior of multi-clustered channels with nonuniform pdf since measurements of practical channels indicated that multipath often arrives in multiple clusters and with non-uniform angular pdf [6,7].

In this paper, we extend Salz-Winters model (single cluster with uniform pdf) to account for more than one cluster and study the effect of clustering with different angular pdfs in details. The emphasis is on analytical analysis, which is further validated using Monte-Carlo simulations. Compact approximate expressions for the correlation coefficient with various angular distributions are derived. A case of two symmetrical clusters is studied in details, including uniform, truncated Gaussian, truncated Laplacian and generic angular pdfs. Using the developed correlation models and the technique presented in $[2,3]$, we study the MIMO channel capacity. In particular, we demonstrate that the angular pdf has negligible effect on the MIMO channel capacity provided that the distribution parameters (i.e. angular spread and mean AOA) are fixed.

\section{Single-Cluster ChanNel Model}

In this model, all the multipath components are assumed to arrive to the $\mathrm{Rx}$ array within $\pm \Delta / 2$ of the mean angle of arrival (AOA) $\varphi$ (see fig. 1) in the horizontal plane (i.e., this is a 2-D model). The AOA probability density function is assumed to be uniform,

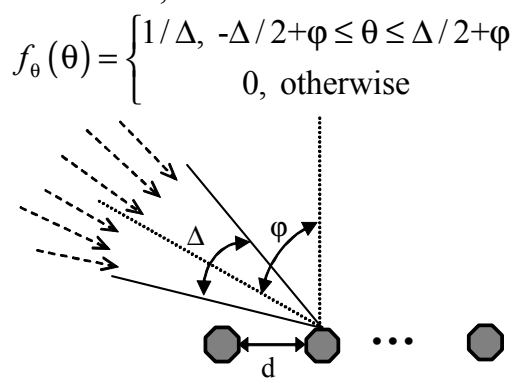

Fig. 1. Incoming multipath signals arrive to the linear antenna array within $\pm \Delta / 2$ of mean angle $\varphi$

In a general case, the normalized correlation between two array elements of a uniform linear array (ULA) can be expressed as

$$
R(z)=\int_{-\pi}^{\pi} f_{\theta}(\theta) \exp [j z \sin \theta] d \theta
$$

where $z=2 \pi d / \lambda, d$ is the element spacing, $\lambda$ is the wavelength, and $j$ is the imaginary unit. Without loss of generality, we further assume that $\lambda=1$. To improve the computational efficiency, a simple but still accurate approximation for small $\Delta<<1$ has been derived using $\sin \beta \approx \beta$ (valid for small $\beta$ ) [3]:

$$
R_{u}(z) \approx \exp (j z \sin \varphi) \operatorname{sinc}(\Delta z \cos \varphi / 2)
$$

where $\operatorname{sinc}(x)=\sin (x) /(x)$. If the AOA pdf is modeled as truncated Gaussian distribution 


$$
f_{\theta}(\theta)=\frac{Q_{g}}{\sqrt{2 \pi} \sigma_{g}} \exp \left(-\frac{(\theta-\varphi)^{2}}{2 \sigma_{g}}\right), \quad-\pi+\varphi \leq \theta \leq \pi+\varphi
$$

where $Q_{g}=1 / \operatorname{erf}\left(\gamma_{g}\right), \gamma_{g}=\pi /\left(\sqrt{2} \sigma_{g}\right)$, the spatial correlation can be approximated as [4]

$$
R_{g}(z) \approx \exp \left(j z \sin \varphi-\left(\sigma_{g} z \cos \varphi\right)^{2} / 2\right)
$$

The angular spread of the uniform pdf is $S_{u}=\Delta /(2 \sqrt{3})$. For truncated Gaussian distribution it is given by

$$
S_{g}=\sigma_{g} \sqrt{1-\left[2 \gamma_{g} \operatorname{erf}\left(\gamma_{g}\right) \cdot \exp \left(\gamma_{g}^{2}\right)\right]^{-1}},
$$

and for small variance $\sigma_{g} \ll 1, \gamma_{g} \gg 1$ and $S_{g} \approx \sigma_{g}$. By setting the same angular spread for both distributions, $S_{u}=S_{g}$ (hence $\sigma_{g} \approx \Delta /(2 \sqrt{3})$ ), it can be shown that (3) and (5) have similar expansion terms [4],

$$
\begin{aligned}
& R_{g}(z)=\left[1-\frac{1}{2}(\sigma z \cos \varphi)^{2}+\frac{1}{8}(\sigma z \cos \varphi)^{4}\right] \exp (j z \sin \varphi) \\
& R_{u}(z)=\left[1-\frac{1}{2}(\sigma z \cos \varphi)^{2}+\frac{3}{40}(\sigma z \cos \varphi)^{4}\right] \exp (j z \sin \varphi)
\end{aligned}
$$

These approximations are correct provided that $\sigma z|\cos \varphi|<1$. As experimental results demonstrate, the AOA pdf is best approximated by the truncated Laplacian distribution [6,7]. Following this observation, we further examine the Laplacian pdf in details. The AOA pdf can be presented as

$$
f_{\theta}(\theta)=\frac{Q_{l}}{\sqrt{2} \sigma_{l}} \exp \left(-\frac{\sqrt{2}|\theta-\varphi|}{\sigma_{l}}\right),-\pi+\varphi \leq \theta \leq \pi+\varphi
$$

where $Q_{L}=1 /\left[1-\exp \left(-\gamma_{l}\right)\right]$ and $\gamma_{l}=\sqrt{2} \pi / \sigma_{l}$. Combining (8) and (2) and using the same approximation as in (3), one obtains

$$
\begin{gathered}
R_{l}(z) \approx \exp (j z \sin \varphi) g(z \cos \varphi) \\
g(x)=\frac{\exp \left(-\gamma_{l}\right)\left[\pi x \cdot \sin (\pi x) / \gamma_{l}-\cos (\pi x)\right]+1}{\left(1-\exp \left(-\gamma_{l}\right)\right)\left[1+\left(\pi x / \gamma_{l}\right)^{2}\right]}
\end{gathered}
$$

for small $\sigma_{l} \ll 1, \gamma_{l} \gg 1$ and $g(x)$ can be further approximated as

$$
g(x) \approx\left[1+\left(\sigma_{l} x\right)^{2} / 2\right]^{-1}
$$

Further, by using the well-known Taylor expansion for $1 /(1+x)$, the first three terms of the approximation (10) are

$$
R_{l}(z) \approx\left[1-\frac{1}{2}\left(\sigma_{l} z \cos \varphi\right)^{2}+\frac{1}{4}\left(\sigma_{l} z \cos \varphi\right)^{4}\right] \mathrm{e}^{j z \sin \varphi}
$$

Note that the angular spread for the Laplacian distribution is

$$
S_{l}=\sigma_{l} \sqrt{1-\frac{e^{-\gamma_{l}}}{1-e^{-\gamma_{l}}}\left(\frac{1}{2} \gamma_{l}^{2}+\gamma_{l}\right)} \approx \sigma_{l} .
$$

For small angular spread $\left(\sigma_{l} \ll 1\right), Q_{l} \approx 1$ and $S_{l} \approx \sigma_{l}$. Hence truncating the distribution's tails to $[-\pi, \pi]$ have negligible effect on the angular spread (as compared to nontruncated distribution). Using the same angular spread for different pdfs, i.e. $S_{u}=S_{g}=S_{l}$ and $\sigma_{l}=\sigma_{g}=\Delta / 2 \sqrt{3}$, and comparing (6), (7) and (11), one can immediately explain the claim in [4] that the approximation (6) is slightly optimistic (lower correlation) when compared to the Laplacian distribution and slightly pessimistic (higher correlation) when compared to the uniform one: this is true because the third term in the (6) is larger than that of (7) and smaller than that of (11).

We conclude that the assumption of uniform angular pdf does not limit significantly the accuracy of the single cluster angular model. As we have shown above, the non-uniform pdf models (Laplacian and truncated Gaussian) produce roughly the same results in terms of the correlation provided that the angular spread is the same for all the distributions. Intuitively, it can be explained by the fact that correlation is an integral of the angular pdf (see (2)). Hence, all the local variations in $f_{\theta}(\theta)$ are smoothed out during the integration and do not affect significantly the final result, i.e. this is a second-order effect. Since system performance is not affected significantly when the correlation is less than 0.5 (this is precisely where the difference between the models is the largest), the performance predictions based on different models will be roughly the same (see Fig. 6). Similar conclusion has also been obtained in [4]. Since uniform distribution is much easier to work with, it should be used for the system performance prediction, which will not result in significant inaccuracy.

\section{TWO-CLUSTER MODEL}

The single cluster channel model has been generalized to multiple clusters [3-5]. In particular, [3] has discussed the two-cluster channel model with uniform pdf in details, including case studies. In order to get some insight into correlation behavior of a multi-cluster channel with different AOA pdfs, let us now study the two-cluster model in more detail.

A. Two symmetric clusters with uniform $p d f$

In this case, the angular pdf can be expressed as

$$
f_{\theta}(\theta)=\left\{\begin{array}{l}
\frac{1}{2 \Delta},\left\{\begin{array}{l}
-\Delta / 2+\varphi_{1} \leq \theta \leq \Delta / 2+\varphi_{1}, \\
-\Delta / 2+\varphi_{2} \leq \theta \leq \Delta / 2+\varphi_{2}
\end{array}\right. \\
0, \text { elsewhere }
\end{array}\right.
$$

The scenario is illustrated in Fig. 2. For two symmetric clusters, $\varphi_{1}=-\varphi_{2}=\varphi$, the spatial correlation is [3],

$$
R(z)=J_{0}(z)+2 \sum_{m=1}^{\infty} J_{2 m}(z) \frac{\cos (2 m \varphi) \sin (m \Delta)}{m \Delta}
$$




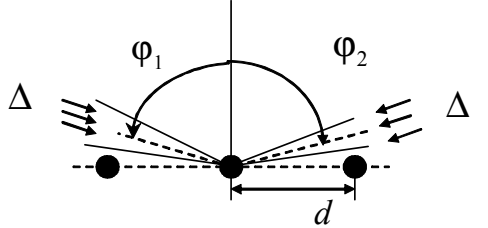

Fig. 2. Incoming multipath is concentrated in two clusters.

and, for small angular spread, can be approximated as

$$
R(z) \approx \cos (z \sin \varphi) \operatorname{sinc}(\Delta z \cos \varphi / 2)
$$

There exist two different types of correlation zero points $R=0$. The element spacing at these points is

$$
d_{0}=\left\{\begin{array}{l}
\frac{m_{1}}{4 \sin \varphi}, m_{1}=1,3,5, \cdots,(2 k-1), \cdots \\
\frac{m_{2}}{\Delta \cos \varphi}, m_{2}=1,2,3, \cdots, k, \cdots
\end{array}\right.
$$

First-type zeros (top) are due to the cosine factor in (14) and the second-type zeros (bottom) are due to the sinc factor. The spacing between first-type zeros is $\Delta d_{01}=1 /(2 \sin \varphi)$ and it does not depend on the angular spread $\Delta$; the zero spacing of the second- type zeros is $\Delta d_{02}=1 /(\Delta \cos \varphi)$ and it does depend on the angular spread - the larger the spread the smaller the spacing. The second- type zeros are the same as for the single cluster model (see eq. 3). The minimum spacing for the first-type zeros is $d_{01, \min }=1 /(4 \sin \varphi)$ and the minimum spacing for the second- type zeros is $d_{02, \min }=1 /(\Delta \cos \varphi)$.

\section{B. Two symmetric clusters with Laplacian $p d f$}

Consider two clusters with equal cluster power allocation and with AOA pdf of each cluster being Laplacian one,

$$
F(\theta)=\frac{1}{2} f_{L}(\theta, \varphi)+\frac{1}{2} f_{L}(\theta,-\varphi)
$$

where $f_{L}(\theta, \varphi)$ is the Laplacian pdf given by (8). Using the same technique as in the previous case, the spatial correlation can be expressed as

$$
R(z) \approx \cos (z \sin \varphi) g(z \cos \varphi)
$$

where $g(z \cos \varphi)$ is approximated by (10) for small angular spread. Comparing (17) and (14), one can see that the approximations for spatial correlation with uniform and Laplacian pdf have the same factor (cosine), which results in oscillations, and different factors that form the envelope: sinc for the uniform pdf and (10) for the Laplacian one. The common factor (cosine) results in the same first type of zeros, located at

$$
d_{0}=\frac{m_{1}}{4 \sin \varphi}, m_{1}=1,3,5, \cdots,(2 k-1), \cdots
$$

Figure 3 compares approximation (17) with numerical results obtained using Monte-Carlo simulation. Clearly, the agreement is good, including zero locations, which is $d_{01, \min }=0.5 \lambda, \Delta d_{01}=\lambda$ in this case. Note also that the second-type zeros are missing in this case, which is consistent with (17) and (10) (since $\mathrm{g}(\mathrm{x})$ is monotonically decreasing function).

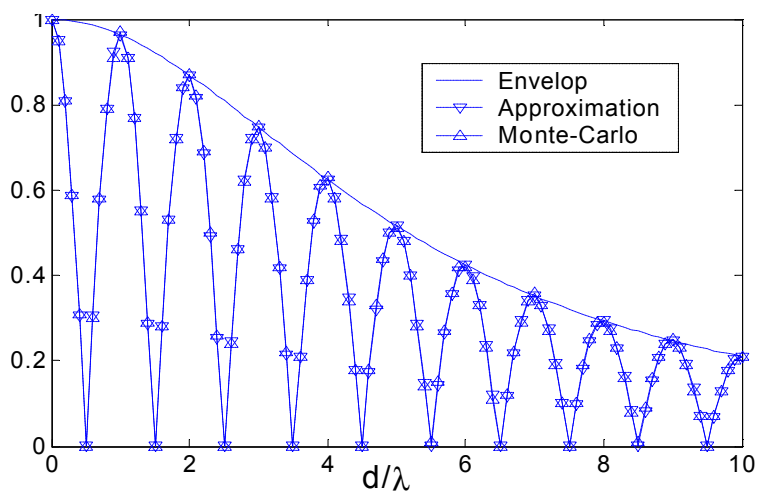

Fig. 3. Correlation coefficient versus element spacing for the case of two symmetric clusters. $\Delta=10^{\circ}, \varphi=30^{\circ}$ with Laplacian pdf

Fig 4 compares correlations predicted by the different models considered above. Clearly, the uniform and Laplacian distributions give the most pessimistic and most optimistic predictions, respectively, which is in the agreement with the arguments above. The Gaussian distribution is "in-between".

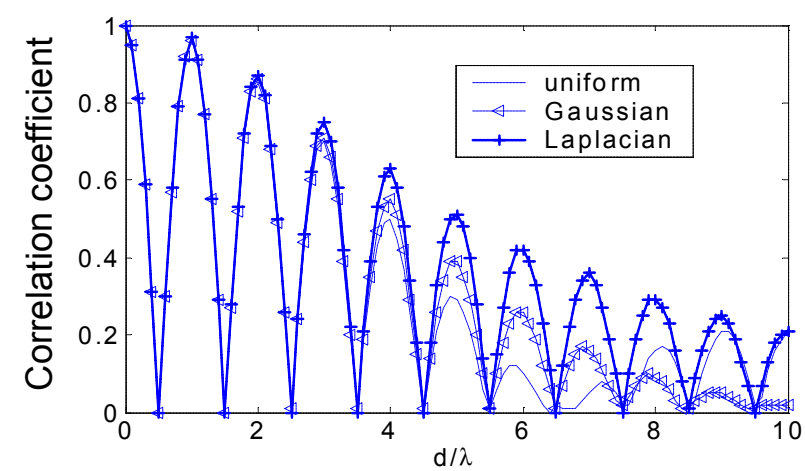

Fig 4. Correlation coefficient versus element spacing for the case of two symmetric clusters. $\Delta=10^{0}, \varphi=30^{\circ}$ with uniform, Gaussian and Laplacian pdf

\section{Two symmetric clusters with general pdf}

Consider a single-cluster model with generic AOA pdf $f(\theta, \varphi)$ and assume that the angular spread is small, i.e. $f(\theta, \varphi)$ has non-negligible values for $\theta \in[\varphi-\Delta, \varphi+\Delta]$ and $\Delta<<1$. Then, (2) can be approximated as

$$
R(z) \approx \exp (j z \sin \varphi) G(z \cos \varphi)
$$

where $G(x)=\int_{-\Delta}^{\Delta} \exp (j x \theta) f(\theta) d \theta$ and $f(\theta, 0)=f(\theta)$. If the angular pdf is symmetrical about the mean AOA, $f(\theta, 0)=f(-\theta, 0)$, then

$$
G(x)=2 \int_{0}^{\Delta} \cos (x \theta) f(\theta) d \theta
$$


Let us now consider the two-cluster case with equal power allocation (per cluster),

$$
F(\theta)=\frac{1}{2} f(\theta-\varphi)+\frac{1}{2} f(\theta+\varphi),
$$

under the same assumptions as above. The correlation is then approximated as

$$
R(z) \approx \cos (z \sin \varphi) \cdot G(z \cos \varphi)
$$

where $\mathrm{G}(\mathrm{x})$ is still given by (19). Comparing (18) and (20), it is clear that the magnitude of (18) is the envelope of (20). Hence, for any AOA pdf two clusters produce oscillations in the correlation due to constructive and destructive addition of their individual contributions (i.e. cosine factor in (20)). Hence, the first-type zeros are the same as for uniform pdf (see (14)); they are not affected by the angular pdf with the clusters. On the contrary, the second-type zeros depend on the angular pdf and may be missing in some cases (i.e. Laplacian and Gaussian distributions).

Good system performance (capacity, diversity gain etc.) requires for low (ideally - zero) correlation. Normally, this is accomplished by setting d large enough. However, as [3] demonstrated, in a two-cluster scenario with uniform pdf the spatial correlation oscillation effect can be beneficially used to achieve low correlation by setting the spacing $d$ at the first type of zeros $d=d_{01, \min }=1 /(4 \sin \varphi)$. Here we generalize this conclusion for any angular distribution in two-cluster symmetric model. Some restrictions may apply for setting antenna spacing to achieve zero correlation in multiple antenna systems [3].

\section{MUlti-Cluster CHANNEL}

It is straightforward to extend the results above to a multicluster model. Assuming equal power allocation for all the clusters, the angular pdf can be presented as

$$
F(\theta)=\frac{1}{N} \sum_{i=1}^{N} f\left(\theta-\varphi_{i}\right)
$$

where $f(\theta)$ is the single-cluster pdf when the mean AOA is zero, and $\varphi_{i}$ is the mean AOA of $i$-th cluster. When angular spread of each cluster is small,

$$
R(z) \approx \frac{1}{N} \sum_{i=1}^{N} e^{j z \sin \varphi_{i}} G\left(z \cos \varphi_{i}\right)
$$

where $G$ is given by (19). In the case of Laplacian cluster pdf, it can be approximated by (10) and in the case of Gaussian cluster pdf it can be approximated by

$$
G(x) \approx \exp \left[-\left(\sigma_{g} x\right)^{2} / 2\right)
$$

\section{MIMO CAPACITY}

Using the approach of [2] and the correlation models above, the MIMO capacity can be evaluated. As an example, we consider a two-cluster channel with 20 i.i.d. multipath components arriving at each cluster; $\varphi_{1}=-30^{\circ}, \varphi_{2}=30^{0}$ (i.e., symmetric). The transmit array elements are assumed to be uncorrelated - all the correlation is concentrated at the $\mathrm{Rx}$ end. The mean capacity of the channel is shown in Fig. 5. Clearly, given the same parameters (i.e. angular spread and the mean AOA), all the different pdfs result in almost the same MIMO capacity. Comparison with the correlation curve indicates that the peaks of correlation correspond to notches of the capacity and vise versa. Also, the capacity oscillations, which do not appear in the single-cluster model [2], are apparent.

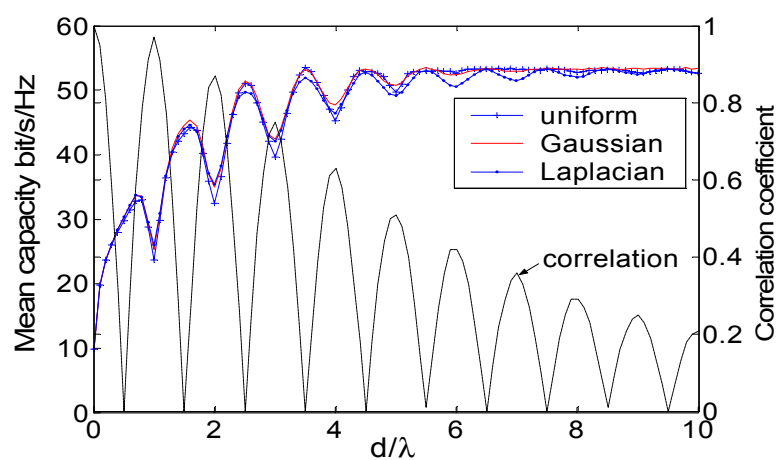

Fig. 5. 10x10 MIMO capacity of a two-cluster channel for various multipath AOA pdf with angular spread $\sigma_{2}=\sigma_{1}=5^{0} / \sqrt{3}, \mathrm{SNR}=20$ $\mathrm{dB} /$ antenna.

\section{REFERENCES}

[1] J. Salz, J.H. Winters, "Effect of Fading Correlation on Adaptive Arrays in Digital Mobile Radio," IEEE Trans. Vehicular Technology, vol. 43, N. 4, pp. 1049-1057, Nov. 1994.

[2] S. Loyka, G. Tsoulos, "Estimating MIMO System Performance Using the Correlation Matrix Approach, " IEEE Communication Letters, v. 6, N. 1, pp. 19-21, Jan. 2002.

[3] G. Zhao, S. Loyka, "Impact of Multipath Clustering on the Performance of MIMO System", IEEE WCNC 2004, Atlanta, Georgia, accepted.

[4] R. M. Buehrer, The Impact of Angular Energy Distribution on Spatial Correlation, IEEE Vehicular Technology Conference, Vancouver, Canada, September 24-28, 2002, pp.1173-1177

[5] L. Schumacher et al, "From Antenna Spacing to Theoretical Capacities - Guidelines for Simulating MIMO Systems," PIMRC 2002, pp. 587-592.

[6] Q. Spencer et al, Modeling the statistical time and angle of arrival characteristics of an indoor multipath channel, IEEE JSAC v.18, N.3, March 2000, pp. 347 - 360.

[7] K. I. Pedersen et al, "A stochastic model of the temporal and azimuthal dispersion seen at the base station in outdoor propagation environments," IEEE Trans. Veh. Tech. , v. 49, No.2, Mar. 2000, pp.437-44 